HPV

\title{
The value of anal cytology and human papillomavirus typing in the detection of anal intraepithelial neoplasia: a review of cases from an anoscopy clinic
}

\author{
P A Fox, J E Seet, J Stebbing, N Francis, S E Barton, S Strauss, T G Allen-Mersh, B G Gazzard, \\ M Bower
}

Sex Transm Infect 2005;81:142-146. doi: 10.1136/sti.2003.008318

See end of article for authors' affiliations

a thes' affilialions

Correspondence to: P A Fox, Department of HIV and Genitourinary Medicine, Chelsea and Westminster Hospital, 369 Fulham Road, London SW10 9NH, UK;

Paul.Fox@eht.nhs.uk

Accepted for publication 19 July 2004

Background: Previous studies have reached differing conclusions about the utility of anal cytology as a screening tool for anal intraepithelial neoplasia (AIN). There is a need also to establish whether HPV typing offers a useful adjunct to screening.

Methods: We analysed data from 99 consecutive homosexual/bisexual male patients (89 HIV-1 positive) who underwent high resolution anoscopy. Follow up visits for these patients were also included, giving a total of 160 anoscopic procedures. Comparison was made between results of anal cytology using the sampling method of Palefsky, and histological findings of biopsies taken from abnormal areas seen on high resolution anoscopic examination of the anal canal. Swabs taken concurrently with the cytology were analysed for the presence of human papillomavirus (HPV) DNA and compared with the cytological and histological findings.

Results: The sensitivity of the cytology was $83 \%$, and the specificity $38 \%$ when compared with histology. At screening of 34 asymptomatic men, $83 \%$ had anal cytological dysplasia and $78 \%$ had AIN. There were no significant differences in the prevalence of hrHPV genotypes between different cytological or histological grades of abnormalities.

Conclusion: Anal cytology by the Palefsky method is simple to undertake, has a sensitivity and specificity comparable with cervical cytology, and can therefore be used as the basis of a pilot screening project in centres with large cohorts of HIV positive homosexual men who have a high risk of developing anal carcinoma. HPV genotyping is not a useful adjunct to cytological screening.

A nal intraepithelial neoplasia (AIN) is usually detected by chance during routine haemorrhoidectomy or excision of anal warts and, because of a potential risk of progression to anal carcinoma, it is considered important to keep under surveillance patients with high grade (AIN 2 or 3 ) lesions. There is an ongoing debate as to whether patients who are at risk should be screened for AIN, concerns being greatest for HIV positive patients.

There has been uncertainty about the value of anal cytology as a screening tool. Two previous UK studies have produced differing conclusions about its utility, whereas data from the Palefsky cohort in San Francisco suggest that anal cytology is indeed worthwhile. We wished therefore to validate the Palefsky method based on a UK population.

An anoscopy clinic was established at the Chelsea and Westminster Hospital in September 2000 primarily for patients with unexplained anal symptoms, suspicious lesions in the anal canal, and those diagnosed with AIN following routine anal surgery. Some asymptomatic patients were also screened because they had fears about anal cancer risk.

\section{METHODS}

Data were collected prospectively from 99 consecutive homosexual/bisexual male patients who underwent high resolution anoscopy. Data from follow up visits on this group of patients were also included.

\section{Cytology}

A Dacron swab was inserted blindly $3 \mathrm{~cm}$ into the anal canal and rotated using a spiral motion, outward pressure was applied on the anal canal and the swab was gradually withdrawn. This is the method developed by Palefsky in San Francisco. The cellular material was smeared onto a glass slide and sprayed immediately with Cytofix (Cell Path) cytological fixative. The slide was stained using the method of Papanicolaou on an automated staining machine. The cellular material was then examined and graded with a system based on the UK criteria established for cervical cytology. ${ }^{1}$ For the purpose of the study it was thought necessary to avoid the "borderline" classification. Smears which did not fulfil the criteria for dyskaryosis, but had parakeratotic cells and mild nuclear changes suggestive of wart virus infection, were classified as "HPV." All slides were reviewed by a single pathologist, without knowledge of the anoscopic findings and without reference to histological findings.

\section{HPV analysis}

The tip of the same Dacron swab was then immersed in $1 \mathrm{ml}$ of sterile phosphate buffered saline (PBS) and sent for human papillomavirus (HPV) analysis. A nested PCR was performed utilising the MY09, MY11, GP5+, and GP6+ primers, as described previously. ${ }^{2}$ The GP5+ primer was fluorophore labelled and the GP6+ primer biotin labelled, in order to generate products for HPV typing. HPV typing was performed by reserve line hybridisation, using 29 HPV DNA sequence specific probes." High risk HPV (hrHPV) was defined as the presence of HPV genotypes 16, 18, 31, 33, $35,39,45,51,52,56,58,59,68,73$, or $82 .{ }^{4}$

Abbreviations: AIN, anal intraepithelial neoplasia; HAART, highly active antiretroviral therapy; HPV, human papillomavirus; hrHPV, high risk HPV; PBS, phosphate buffered saline; PYAR, person years at risk 


\section{Anoscopy}

With the patient lying in the left lateral position on a specially modified examination couch, the anal canal was palpated using $2 \%$ lidocaine gel as a lubricant. A transparent proctoscope was then inserted, and through it a Dacron swab wrapped in gauze and soaked with $3 \%$ acetic acid was introduced. The proctoscope was then removed, and after 2 minutes the swab was removed and the proctoscope reinserted. The anal canal was visualised using a Zeiss colposcope, concentrating on the squamocolumnar junction at the dentate line and the transformation zone which lies immediately proximal to it. The aceto-white areas of AIN have very similar appearances to CIN when seen through the colposcope. ${ }^{5}$ The area or areas which were suggestive morphologically of the highest grade of AIN were biopsied using rectoscopy forceps. Samples were fixed in 10\% neutral buffered formalin and sent for histological examination.

\section{Histology}

The samples were embedded in paraffin wax and sections of $5 \mu \mathrm{m}$ were stained with haematoxylin and eosin. Not less than three sections were examined from each biopsy and all were reviewed by a single pathologist. AIN was graded using the criteria routinely used for CIN, based on the proportion of the epithelium occupied by basaloid undifferentiated cells. In AIN 1 these cells are confined to the lower third of the epithelium, while in high grade AIN, they occupy the lower to middle third (AIN 2) or the middle third to full thickness (AIN 3). Where the AIN was on the borderline between two grades it was counted as belonging to the higher grade.

\section{Statistical analysis}

Incidence figures were calculated by, firstly, generating person days of follow up which were converted to person years at risk (PYAR). PYAR was estimated from entry into the cohort to either end of study period, the development of anal cancer, the last recorded visit or if the patient had died during their follow up then their death date. In order to keep the coefficient of the PYAR constant, this was $\log _{10}$ transformed and used as the offset in the Poisson regression. The data were analysed using the Genmod procedure in SAS version 9.0 (Cary, NC, USA) with $\log _{\mathrm{e}}$ link and Poisson error distributions.

The significance between the groups was analysed using the Kruskal-Wallis non-parametric one way test of variance using the SAS software. This determined whether any group showed a statistically significant difference from others.

Between group comparisons of quantitative data with hypergeometric distributions were analysed using the Kruskal-Wallis test. Qualitative data are presented as proportions. Logistic regression analysis was used to identify factors associated with positive cytology and histology. Data were analysed using SAS software. All p values quoted are two tailed.

\section{RESULTS}

One hundred and sixty anoscopic procedures were reviewed from 99 homosexual or bisexual men in whom both cytological and histological examinations were planned but including $17(10 \%)$ procedures where no biopsy was performed because the patient was either anoscopically normal, or only a few exophytic warts were present. Eighty nine $(90 \%)$ patients were HIV positive and 10 were known to be HIV negative. Patients were referred to the anoscopy clinic for one of three reasons. Thirty four (34\%) patients were asymptomatic referrals for screening, of whom 11 of these had ongoing anal warts and two had penile intraepithelial neoplasia. Twenty seven (27\%) patients were referred with troublesome anal symptoms: nine with bleeding, 12 with chronic soreness and irritation (six of these 12 also had some warts in the anal canal), two with anal pain of unknown origin, three with suspicious perianal lesions, and one patient had felt a small plaque in the canal. Thirty eight $(38 \%)$ patients were referred with previously diagnosed AIN which was found during routine surgery for excision of warts, polyps, skin tags, and haemorrhoids. A subgroup of five of these 38 patients had a history of both AIN and treated invasive squamous carcinoma of the anus.

The cytological findings revealed normal cytology or HPV changes in only $41 / 160$ (26\%) examinations; mild dyskaryosis in $61(38 \%)$, moderate dyskaryosis in $34(21 \%)$, and severe dyskaryosis in 24 (15\%). Histological biopsies of suspicious aceto-white areas were available from 142 procedures. The histological findings were normal in five $(4 \%)$, features of HPV infection only in $17(12 \%)$, AIN 1 in $26(18 \%)$, AIN 2 in $53(37 \%)$, AIN 3 in 37 (26\%), inflammatory changes in three $(2 \%)$, and ulceration only in one $(1 \%)$.

Simultaneous cytological and histological examinations were available for 141 anoscopic procedures and the sensitivity, specificity, and predictive values of blind cytological examination was calculated by comparison of the histological examination of anoscopically guided biopsies. Positive results were defined as cytological appearances of dyskaryosis of any grade and histological findings of AIN of any grade. In 95 procedures cytology and histology were concordantly positive; in 16 procedures cytology was abnormal but biopsy showed no AIN; in 20 procedures cytology was negative or revealed HPV only but AIN was detected histologically, and in 10 procedures cytology and histology were concordantly negative. This gives a sensitivity for cytology of $83 \%$ and the specificity is $38 \%$, while the positive predictive value is $86 \%$ (95\% confidence interval: 78 to 91 ) and the negative predictive value is $33 \%$ (95\% confidence

\begin{tabular}{l}
$\begin{array}{l}\text { Table } 1 \\
\text { the HIV positive group }\end{array}$ \\
\begin{tabular}{lll} 
Correlation between histological and cytological findings and CD4 cell counts in \\
& $\begin{array}{l}\text { Median CD4 cell count } \\
\text { (cells } \times 10^{6} / \text { /I) (range) }\end{array}$ & $\begin{array}{l}\text { Kruskall Wallis } \\
\text { p value }\end{array}$ \\
\hline AIN & $280(60-559)$ & \\
No AIN & $474(125-768)$ & 0.12 \\
AIN 1 & $329(16-1035)$ & \\
AIN 2 & $365(37-700)$ & \\
AIN 3 & $389(60-780)$ & \\
Dyskaryosis & $328(16-700)$ & \\
No dyskaryosis & $340(144-507)$ & \\
Mild dyskaryosis & $358(37-1035)$ & \\
Moderate dyskaryosis & & \\
Severe dyskaryosis &
\end{tabular} \\
\hline
\end{tabular}


Table 2 Oncogenic HPV types isolated in relation to abnormality detected by anoscopy and cytology

\begin{tabular}{|c|c|c|c|c|c|c|c|c|c|c|c|c|c|c|c|c|c|}
\hline HPV type & No & 16 & 18 & 31 & 33 & 35 & 39 & 45 & 51 & 52 & 56 & 58 & 59 & No HPV & 1 types & 2 types & $>2$ types \\
\hline \multicolumn{18}{|l|}{ Histology } \\
\hline Normal histology & 18 & 8 & 5 & 3 & 6 & 1 & 0 & 3 & 2 & 1 & 1 & 4 & 3 & 0 & 5 & 5 & 8 \\
\hline AIN 1 & 19 & 12 & 3 & 0 & 5 & 0 & 0 & 4 & 1 & 0 & 0 & 2 & 0 & 0 & 6 & 6 & 1 \\
\hline AIN 2 & 45 & 28 & 9 & 8 & 11 & 6 & 5 & 8 & 1 & 0 & 2 & 11 & 3 & 2 & 9 & 9 & 20 \\
\hline AIN 3 & 29 & 20 & 10 & 6 & 7 & 2 & 4 & 4 & 2 & 0 & 1 & 5 & 1 & 0 & 8 & 6 & 8 \\
\hline \multicolumn{18}{|l|}{ Cytology } \\
\hline Cytology normal & 29 & 11 & 8 & 5 & 3 & 2 & 0 & 5 & 1 & 0 & 1 & 3 & 2 & 0 & 9 & 12 & 8 \\
\hline Mild dyskaryosis & 49 & 30 & 10 & 6 & 11 & 1 & 4 & 11 & 2 & 1 & 1 & 10 & 4 & 1 & 13 & 13 & 22 \\
\hline Moderate dyskaryosis & 28 & 19 & 10 & 6 & 13 & 3 & 4 & 6 & 1 & 0 & 1 & 9 & 1 & 0 & 2 & 4 & 22 \\
\hline Severe dyskaryosis & 20 & 13 & 2 & 3 & 1 & 3 & 1 & 1 & 2 & 0 & 2 & 2 & 0 & 1 & 10 & 3 & 6 \\
\hline
\end{tabular}

interval: 19 to 51 ). The likelihood ratio for a positive test was 1.3 and for a negative test was 0.45 .

The median CD4 cell count at the time of biopsy in patients with HIV in each of the histological groups is shown in table 1 . There was no correlation between histological grade and CD4 cell count (Kruskall-Wallis $p=0.12$ ). Similarly there was no correlation between severity of cytological findings and CD4 cell count (Kruskall-Wallis $\mathrm{p}=0.61$ ).

Genotype analysis for HPV was available for 126 procedures including 29 with no cytological dyskaryosis (table 2). Simultaneous histology from biopsies was available for 111 procedures including 18 without AIN. HPV-16 genotype was isolated in 73 of the 126 (58\%) cytological samples including $11 / 29(38 \%)$ without dyskaryosis. There were no significant associations between histological or cytological grade and the presence of any hrHPV genotype (see table 3).

Univariate logistic regression analysis showed that neither HIV serostatus $(p=0.56)$, CD4 cell count quartile $(p=0.28)$, nor the presence of HPV $16(p=0.41)$ were associated with positive anal cytology. Similarly, neither HIV serostatus $(p=0.32), \quad C D 4$ cell count quartile $(p=0.89)$, nor the presence of HPV $16(p=0.88)$ were associated with positive anal histology.

Although detailed evaluation of screening of asymptomatic patients is beyond the scope of this analysis, the cohort included 34 asymptomatic patients (including three HIV negative homosexual men) and 46 cytological screens and 41 anoscopic biopsies were performed on these patients. Thirty eight of 46 $(83 \%)$ cytological samples demonstrated dyskaryosis, including $10(22 \%)$ with moderate dyskaryosis and four $(9 \%)$ with severe dyskaryosis. These high rates of anal pathology in asymptomatic patients were recapitulated in the biopsy results. Thirty two of $41(78 \%)$ samples demonstrated AIN, and AIN 2 was present in $21(51 \%)$ and AIN 3 in three (7\%).

\section{DISCUSSION}

The overall incidence of invasive anal cancer at the Chelsea and Westminster Hospital cohort of 8640 HIV-l seropositive patients diagnosed since 1986 (40 126 patient years of follow up) is $60 / 10^{5}$ patient years ( $95 \%$ confidence intervals (CI): 40 to 89). This compares with an incidence of 0.52 (95\% CI: 0.27 to 0.78$) / 10^{5}$ patient years in the age and sex matched general population of south east England. Moreover, the incidence of invasive anal cancer in the HIV positive cohort has not declined since the era of highly active antiretroviral therapy (HAART). The incidence was 35 (95\% confidence interval (CI): 15 to 72$) / 10^{5}$ patient years follow up in the pre-HAART era and is 92 (95\% CI: 52 to 149$) / 10^{5}$ patient years follow up in the post-HAART era. ${ }^{6}$ It was hoped that HAART might have a role in the resolution of high grade AIN but it is becoming evident that this is not the case, as $75 \%$ of men treated with HAART experienced no regression of AIN 2 or $3 .^{7}$

In this cohort a significant problem has been the relatively advanced stage of disease at presentation, with $38 \%$ having T3 or T4 disease, $31 \%$ having nodal disease and $6 \%$ presenting with distant metastases. This late presentation can be explained in part by the attribution, in the clinical setting, of anal symptoms to the presence of warts or haemorrhoids. A commonly held belief within genitourinary medicine is that anal warts have no significance and do not necessarily need to be treated. This has not helped homosexual men to be aware of the potential seriousness of a lump in the anus. The overall actuarial survival at 2 years for patients with invasive anal cancer is $47 \%$ (95\% CI: 24 to 70 ). ${ }^{6}$ Early intervention with chemoradiotherapy is associated with a better outcome, but the early detection of lesions poses substantial difficulties. The detection of pre-invasive anal lesions and invasive anal cancer at early stages of disease could substantially improve survival.

The prevalence of AIN in HIV positive homosexual and bisexual male cohorts has been found to be as high as 36\% in San Francisco: $31 \%$ with low grade dysplasia and $4.9 \%$ with high grade. ${ }^{8}$ The same study demonstrated a CD4 stratification, with high grade AIN being present in $3.4 \%$ of patients with $\mathrm{CD} 4$ over $500 \times 10^{6} / \mathrm{l}$ rising to $8.7 \%$ of those with CD4 under $200 \times 10^{6} /$ l. A slightly lower AIN prevalence of $26 \%$ was

\begin{tabular}{llll} 
Table 3 & HPV genotype analysis according to anal cytological and histological findings \\
\hline & HPV-16 & Any hrHPV & $\begin{array}{c}\text { Two or more hrHPV } \\
\text { genoptypes present }\end{array}$ \\
\hline No dyskaryosis & $11 / 29(38 \%)$ & $21 / 29(72 \%)$ & $8 / 29(28 \%)$ \\
Mild dyskaryosis & $30 / 49(61 \%)$ & $37 / 49(76 \%)$ & $20 / 49(41 \%)$ \\
Moderate dyskaryosis & $19 / 28(68 \%)$ & $18 / 28(64 \%)$ & $15 / 28(54 \%)$ \\
Severe dyskaryosis & $13 / 20(65 \%)$ & $12 / 20(60 \%)$ & $6 / 20(30 \%)$ \\
p Value & $p=0.06$ & $p=0.9$ & $p=0.12$ \\
No AIN & $8 / 18(44 \%)$ & $12 / 18(67 \%)$ & $7 / 18(39 \%)$ \\
AIN 1 & $12 / 19(63 \%)$ & $15 / 19(79 \%)$ & $6 / 19(32 \%)$ \\
AIN 2 & $28 / 45(62 \%)$ & $33 / 45(73 \%)$ & $22 / 45(49 \%)$ \\
AIN 3 & $20 / 29(69 \%)$ & $17 / 29(59 \%)$ & $11 / 29(38 \%)$ \\
p Value & $p=0.41$ & $p=0.35$ & $p=0.29$ \\
\hline
\end{tabular}


found in the Seattle cohort study. ${ }^{9}$ It has been estimated that the risk of developing high grade AIN in HIV positive men is similar to risk of CIN 2-3 in HIV negative female STD patients. ${ }^{10}$ Among the 41 samples from asymptomatic patients in this study, the rates of AIN were remarkably high at $78 \%$. Moreover, within this cohort $58 \%$ had high grade AIN (grade 2 or 3 ). This very high prevalence is probably the result of a degree of selection bias, in that clinicians were referring the patients whom they perceived to be at highest risk. In contrast with the San Franscisco study, we found no significant correlation between CD4 cell count and the presence of anal dyskaryosis or AIN.

The incidence of invasive anal carcinoma of $60 / 10^{5}$ patient years (95\% confidence interval: 40-89) among patients attending the Chelsea and Westminster Hospital is higher than has been reported elsewhere. ${ }^{11}$ By comparison, the risk of invasive anal cancer in homosexual men before the advent of HIV in the United States was $12.5-36.9$ per $10^{5}$ patient years. ${ }^{12}$ In the general population anal cancer is a disease of the elderly and the UK national incidence in males under the age of 50 between 1984 and 1993 was 0.3 per 100000 per annum. ${ }^{13}$ The high incidence, relatively advanced stage at diagnosis, and poor outcome have all been impetuses for the development of a preventative strategy in this patient group. The recognition of AIN as the likely precursor lesion and the ability to detect this has encouraged clinicians to explore the possibility of screening for pre-invasive anal disease in order to reduce the mortality of anal cancer.

Here, we report that a sensitivity of $83 \%$ and specificity of $38 \%$ for anal cytology compared to histology from anoscopically directed biopsy. The high prevalence of high grade lesions in our clinic population, with $63 \%$ of biopsies demonstrating AIN 2 or 3, may have resulted in a higher sensitivity, and possibly in a lower specificity than might be expected from a more representative cohort. In the San Franscico cohort study of 265 HIV positive homosexual men $4 \%$ had histologically confirmed high grade AIN; sensitivity of anal cytology using the same Palefsky method was reported as being $69 \%$, rising to $81 \%$ for subsequent visits, with a specificity of $59 \% .{ }^{14}$ In the Manchester study 38 HIV positive homosexual men had both cytological and histological examinations using a similar technique except that the squamo-columnar junction was visualised with a proctoscope before obtaining the sample for cytology. In this cohort the prevalence of high grade dyskaryosis was $10 \%$, and the sensitivity of the anal cytology increased steadily with repetition to $78 \%$, with a specificity of $82 \%$ for high grade AIN. ${ }^{15}$ However, focused cytological sampling with this direct visualisation technique is sufficiently technically difficult to make it unsuitable for a large scale screening programme. In another London centre, de Ruiter et al compared the use of a cytobrush to perform cytology with anoscopy and biopsy. ${ }^{16}$ She reported a sensitivity of $34 \%$ and specificity of $72 \%$, yielding a positive predictive value of $41 \%$ and a negative predictive value of $66 \%$. The patients in this study were recruited from a genitourinary medicine clinic, and $78 \%$ had anal warts. The presence of warts in the canal probably accounts for the reduced cytological detection of dysplasia for two reasons: firstly, the dysplastic areas may lie underneath the exophytic warts and not be sampled adequately; and, secondly, they may be difficult to visualise on a slide covered with keratin and parakeratotic debris from the surface of the lesions. Anoscopy itself can be quite unsatisfactory in patients with extensive anal warts, and it is advisable for these to be treated before anoscopy is undertaken, by surgical excision if necessary, especially if there are any concerns about possible malignancy.

It is apparent that anoscopy and biopsy is an imperfect gold standard for determining the sensitivity and specificity of the cytology, as there will be occasions when a small area of dysplasia is missed visually because it lies hidden in a fold of mucosa or has not stained adequately. Areas of AIN are usually readily distinguishable from flat warts by the absence of villi, but on occasion the two can be indistinguishable. A few patients with repeatedly abnormal cytology have remained anoscopically normal, with no detectable hrHPV.

There is a strong and consistent link between HPV infection and anal cancer. One line of evidence to support this is provided by the research of Frisch and colleagues, who isolated HPV 16 DNA from $87 \%$ of 386 anal tumour samples, with HPV 18 in 7\%, and HPV 33 in $6 \% .{ }^{17}$ In our study, 58\% had detectable HPV 16 genotype present, $70 \%$ had a high risk HPV genotype present, and 39\% had two or more high risk HPV genotypes present. Despite these high rates, there was no correlation between cytological atypia or intraepithelial neoplasia and the presence of these HPV genotypes. Thus, the presence or absence of HPV 16 and other high risk HPV genotypes was a poor determinant of likelihood of having high grade AIN in this predominantly HIV positive group. Such testing does not therefore appear to have much potential as a screening tool.

The demonstration that blind anal cytological screening is straightforward and associated with adequate sensitivity and specificity in a cohort at high risk of AIN suggests that this technique could form the basis of a screening protocol.

Screening patients for AIN has significant cost implications, and this has deterred any clinic in the United Kingdom from setting up a true screening programme. There are three reasons why a screening programme might be beneficial: firstly, patients with high grade AIN would be made aware of a potential risk of anal carcinoma, and might be more likely to report an anal lump at an earlier, more treatable stage; secondly, careful follow up would detect some early anal carcinomas at a treatable stage; and, thirdly, such a cohort is needed in order to develop effective treatments. It would be advisable to screen all HIV positive patients: there is an as yet unconfirmed report that heterosexual male injecting drug users have a high prevalence of AIN. ${ }^{18}$

In order to establish a pilot screening programme it is essential to be clear on the appropriate management for each outcome. We suggest that there is no compelling reason to treat low grade AIN, which should be monitored with annual cytology. For small high grade AIN lesions that are few in number excision or ablation might offer the prospect of a cure. This might be undertaken by the anoscopist without anaesthesia using trichloroacetic acid, ${ }^{19}$ or by excision or cauterisation in the operating theatre. For large or multiple lesions such an approach is much more problematical, and the morbidity from surgical treatments is high, with uncontrolled pain for 2.9 weeks after the procedure in 50\% of patients. ${ }^{19}$ This matters because the recurrence rate in HIV positive patients is very high at $79 \%$ within a mean 28 month follow up period. ${ }^{19}$ For large or multiple lesions we therefore propose monitoring with 6 monthly anoscopy, which would enable any carcinoma to be identified at a relatively early stage, at which time local excision followed by combined chemotherapy and radiotherapy is an effective treatment for anal squamous carcinoma. ${ }^{20}$ An alternative treatment for widespread high grade AIN might be complete anal mucosectomy. ${ }^{21}$ These approaches are clearly not ideal, but in the long term more acceptable treatments-topical, immunomodulatory, or surgical-can only be developed if there is a cohort of patients with high grade disease.

\section{ACKNOWLEDGEMENTS}

We wish to thank the following: Sundhiya Mandalia for her statistical advice and help; Joel Palefsky's team in San Francisco for teaching PF how to take anal cytology and to perform high resolution anoscopy (supported by a grant form St Stephen's AIDS 
Trust); all the nurses in the John Hunter Clinic for their assistance during the procedures, with special thanks to Helen Selby, Paul Radcliffe, and Lovelle Smith; the colleagues from various hospitals who have referred patients to the clinic, and above all the patients themselves who have endured much and complained little.

\section{CONTRIBUTORS}

$\mathrm{SB}, \mathrm{TA}-\mathrm{M}, \mathrm{NF}, \mathrm{MB}, \mathrm{PF}$, and $\mathrm{BG}$ contributed in various ways to the initiation of the project; PF saw and examined all the patients; JES and NF performed the cytological and histological analyses; SS performed the HPV analysis; JS, MB, and PF analysed and interpreted the results; $\mathrm{PF}$ and $\mathrm{MB}$ wrote the paper; and all authors have reviewed and approved the final version of the paper.

\section{Authors' affiliations}

P A Fox, S E Barton, B G Gazzard, Department of HIV and Genitourinary Medicine, Chelsea and Westminster Hospital, 369 Fulham Road, London SW10 9NH, UK

J E Seet, N Francis, Department of Histopathology, Department of Histopathology, Charing Cross Hospital, Hammersmith Hospitals NHS Trust, London W6 8RF, UK

J Stebbing, M Bower, Department of Oncology, Chelsea and Westminster Hospital, London, UK

S Strauss, HPV Reference Laboratory, Health Protection Agency, 61 Colindale Avenue, London NW9 5HT, UK

T G Allen-Mersh, Department of General Surgery, Chelsea and Westminster Hospital, London, UK

\section{REFERENCES}

1 Evans DM, Hudson EA, Brown CL, et al. Terminology in gynaecological cytopathology: report of the Working Party of the British Society for Clinical Cytology. J Clin Pathol 1986;39:933-44. Endorsed by NHS cervical screening programme 1995 and 2000.

2 Strauss S, Sastry P, Sonnex C, et al. Contamination of environmental surfaces by genital human papillomaviruses. Sex Transm Infect 2002;78:135-8.

3 Jordens JZ, Lanham S, Pickett MA, et al. Amplification with molecular beacon primers and reverse line blotting for the detection and typing of human papillomaviruses. J Virol Methods, 2000;89:29-37.

4 Munoz N, Bosch FX, de Sanjose S, et al. Epidemiologic classification of human papillomavirus types associated with cervical cancer. N Engl J Med 2003;348:518-27.
5 Jay N, Berry JM, Hogeboom CJ, et al. Colposcopic appearance of anal squamous intraepithelial lesions: relationship to histopathology. Dis Colon Rectum 1997;40:919-28.

6 Bower M, Powles T, Newsom-Davis T, et al. HIV associated anal cancer-has HAART reduced the incidence or improved the outcome? J Acquir Immune Defic Syndr 2004; (in press).

7 Palefsky JM. Anal squamous intraepithelial lesions: relation to HIV and human papillomavirus infection. J Acquir Immune Defic Syndr 1999;21(Suppl 1):S42-8.

8 Palefsky JM, Holly EA, Ralston ML, et al. Anal squamous intraepithelial lesions in HIV-positive and HIV-negative homosexual and bisexual men: prevalence and risk factors. J Acquir Immune Defic Syndr Hum Retrovirol 1998; 17:320-6.

9 Kiviat NB, Critchlow CW, Holmes KK, et al. Association of anal dysplasia and human papillomavirus with immunosuppression and HIV infection among homosexual men. AIDS 1993;7:43-9.

10 Critchlow CW, Surawicz CM, Holmes KK, et al. Prospective study of high grade anal squamous intraepithelial neoplasia in a cohort of homosexua men: influence of HIV infection, immunosuppression and human papillomavirus infection. AIDS 1995;9:1255-62.

11 Goedert JJ, Cote TR, Virgo P, et al. Spectrum of AIDS-associated malignant disorders. Lancet 1998;351:1833-9.

12 Daling JR, Weiss NS, Klopfenstein LL, et al. Correlates of homosexual behavior and the incidence of anal cancer. JAMA 1982;247:1988-90.

13 Office for National Statistics. Codes ICD9 154.2 and 154.3. London: Data from UK Office for National Statistics Cancer Intelligence Database.

14 Palefsky JM, Holly EA, Hogeboom CJ, et al. Anal cytology as a screening tool for anal squamous intraepithelial lesions. J Acquir Immune Defic Syndr Hum Retrovirol 1997; 14:415-22.

15 Lacey HB, Wilson GE, Tilston P, et al. A study of anal intraepithelial neoplasia in HIV positive homosexual men. Sex Transm Infect 1999;75:172-7.

16 De Ruiter A, Carter P, Katz DR, et al. A comparison between cytology and histology to detect anal intraepithelial neoplasia. Genitourin Med 1994:70:22-5

17 Frisch M, Fenger C, van den Brule AJ, et al. Variants of squamous cell carcinoma of the anal canal and perianal skin and their relation to human papillomaviruses. Cancer Res 1999:59:753-7.

18 Piketty C, Darragh TM, Da Costa M, et al. High prevalence of anal human papillomavirus infection and anal cancer precursors among HIV infected persons in absence of anal intercourse. Ann Intern Med 2003;138:453-9.

19 Chang GJ, Berry JM, Jay N, et al. Surgical treatment of high-grade anal squamous intraepithelial lesions: a prospective study. Dis Colon Rectum 2002:45:453-8.

20 Cleator S, Fife K, Nelson M, et al. Treatment of HIV-associated invasive anal cancer with combined chemoradiation. Eur J Cancer 2000;36:754-8.

21 Lyons M, Francis N, Allen-Mersh TG. Treatment of grade 3 anal intraepithelial neoplasia by complete anal mucosal excision without fecal diversion: report of a case. Dis Colon Rectum 1999;42:1342-4. 